'Instituto de Anatomía, Histología y Patología, Facultad de Medicina, Universidad Austral de Chile. Valdivia, Chile. Instituto de Farmacia, Facultad de Ciencias, Universidad Austral de Chile, Valdivia, Chile.

${ }^{3}$ Grupo IRyS. Escuela de Educación Física. Pontificia Universidad Católica de Valparaíso. Valparaíso, Chile.

${ }^{4}$ Departamento de Educación Física. Facultad de Educación. Universidad de Concepción. Concepción, Chile. ${ }^{5}$ Departamento de Ciencias de la Actividad Física, Universidad de Los Lagos. Osorno Chile.

${ }^{6}$ Grupo de Investigación Calidad de Vida y Estilos de Vida Saludable, Universidad del Biobío. Chillán, Chile.

${ }^{7}$ Departamento de Educación Física, Deportes y Recreación, Universidad de la Frontera, Temuco, Chile.

${ }^{8}$ Human Nutrition Research Centre, Institute of Cellular Medicine, University of Newcastle. Newcastle upon Tyne, United Kingdom. ${ }^{9}$ BHF Glasgow Cardiovascular Research Centre, Institute of Cardiovascular and Medical Science, University of Glasgow. Glasgow, United Kingdom. *AML y MAM contribuyeron de igual forma a este manuscrito y deben ser consideradas como primer autor compartido. ${ }^{a}$ Magíster Neurociencias y Salud Mental. ${ }^{b}$ Magíster Nutrición y Dietética.

'Doctor en Ciencias de la Actividad Física y Deportes ¿Magíster en Educación Física. eDoctor en Ciencias de la Salud. fMagíster en Educación. 9 Doctor en Estudios del Movimiento Humano

hDoctor en Ciencias Cardiovasculares y Biomédicas.

Fuente de apoyo financiero: Este estudio fue financiado por la beca de doctorado CONICYT otorgada a CCM (2007-2011).

Recibido el 30 de mayo de 2016, aceptado el 4 de abril de 2017

Correspondencia a:

Dr. Carlos Celis-Morales BHF Glasgow Cardiovascular Research Centre 126 University Avenue, Glasgow University, Glasgow, United Kingdom G12 8TA carlos.celis@glasgow.ac.uk

\section{El sedentarismo se asocia a un incremento de factores de riesgo cardiovascular y metabólicos independiente de los niveles de actividad física}

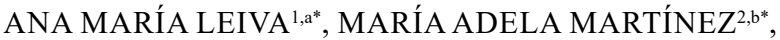 \\ CARLOS CRISTI-MONTERO ${ }^{3, \mathrm{c}}$, CARLOS SALAS ${ }^{4, \mathrm{~d}}$, \\ RODRIGO RAMÍREZ-CAMPILLO ${ }^{5, \mathrm{e}}$, XIMENA DÍAZ MARTÍNEZ ${ }^{6, \mathrm{f}}$, \\ NICOLÁS AGUILAR-FARÍAS ${ }^{7, g,}$ CARLOS CELIS-MORALES $8,9, \mathrm{~h}$ \\ Sedentary lifestyle is associated with \\ metabolic and cardiovascular risk factors \\ independent of physical activity
}

Background: Sedentary behavior is a main risk factor for cardiovascular disease and mortality. Aim: To investigate the association between sedentary behavior and metabolic and cardiovascular risk factors. Material and Methods: We assessed 322 participants aged between 18 to 65 years. Physical activity and sedentary behavior were measured with accelerometers (Actigraph ${ }^{\varpi}$ ). Body mass index (BMI), waist circumference, percentage of body fat, diet and blood markers (glucose, lipid profile, insulin and HOMA-IR) were measured with standardized protocols. Results: Thirty four percent of participants were physically inactive and spent on average $8.7 \mathrm{~h} /$ day on sedentary activities. Per one hour increase in sedentary behavior there were significant adverse changes in glucose (4.79 mg/dl), insulin (2.73 pmol/l), HOMA-IR (0.75), BMI (0.69 kg/ $\left.\mathrm{m}^{2}\right)$, waist circumference $(1.95 \mathrm{~cm})$, fat mass $(1.03 \%)$, total cholesterol ( $9.73 \mathrm{mg} / \mathrm{dl}), \mathrm{HDL}$-cholesterol $(-3.50 \mathrm{mg} / \mathrm{dl}), \mathrm{LDL}$-cholesterol $(10.7 \mathrm{mg} /$ dl) and triglycerides $(12.4 \mathrm{mg} / \mathrm{dl})$. These findings were independent of main confounding factors including total physical activity, dietary factors, BMI and socio-demographics. Conclusions: The detrimental effect of sedentary behaviors on cardiometabolic and obesity-related traits is independent of physical activity levels. Therefore, reducing sedentary time should be targeted in the population apart from increasing their physical activity levels.

(Rev Med Chile 2017; 145: 458-467)

Key words: Cardiovascular Diseases; Metabolic Diseases; Obesity; Risk Factors; Sedentary Lifestyle. 
$\mathrm{D}$ urante la última década, el sedentarismo (principalmente tiempo destinado a estar sentado) ha emergido como un importante factor de riesgo vinculado al desarrollo de obesidad, diabetes mellitus tipo 2 (DMT2), hipertensión arterial, síndrome metabólico, enfermedades cardiovasculares (ECVs), cáncer y mortalidad ${ }^{1,2}$.

El sedentarismo se define como actividades asociadas a un gasto energético $<1,5$ METs (MET $=$ equivalente metabólico basal; 1 MET $=\sim 3,5 \mathrm{mlO} / \mathrm{kg} / \mathrm{min}$ ) e incluye actividades como estar sentado, ver televisión, conducir, entre otras $^{3}$. A nivel global, se estima que entre $55 \%$ y $70 \%$ de las actividades que se realizan diariamente ( $\sin$ considerar el tiempo destinado a dormir) son de tipo sedentarias ${ }^{1,4-6}$. A nivel nacional, los chilenos han experimentado cambios importantes en sus estilos de vida durante las últimas tres décadas, generando que actualmente 19,8\% de la población sea físicamente inactiva ${ }^{7}$. Además, $35,9 \%$ de la población destina $>4 \mathrm{~h}$ al día a estar sentado, incrementando las posibilidades de perjudicar su salud cardiovascular ${ }^{8,9}$. Estudios que han descrito niveles de sedentarismo en población chilena, han cuantificado el tiempo sedente usando cuestionarios, por lo que dichos resultados podrían no representar la verdadera relación que existe entre sedentarismo y marcadores de salud, debido a la sobreestimación a la cual se asocian la medición de actividad física (AF) a través de cuestionarios ${ }^{10,11}$. En consecuencia, el objetivo de este estudio fue investigar la asociación entre el nivel de sedentarismo -medido por acelerometría- con factores de riesgo cardiovascular y metabólicos asociados a la obesidad.

\section{Material y Método}

\section{Diseño del estudio}

Estudio observacional analítico de corte transversal que utiliza información del proyecto GENADIO (Genes, Ambiente, Diabetes y Obesidad) realizado entre los años 2009 y 2011 en Chile, que evaluó la prevalencia de factores de riesgo de ECVs y contó con la aprobación de los comités de ética de las universidades de Chile, Concepción y Glasgow (Reino Unido). Todos los participantes firmaron un consentimiento informado.

La muestra quedó conformada por 322 personas de las regiones del Biobío y Los Ríos. El cálculo del tamaño muestral se estimó en base a diferencias en HOMA-IR entre grupos étnicos, y ha sido descrito en extenso en publicaciones previas ${ }^{8}$. Los criterios de exclusión fueron: (a) personas menores a 18 años; (b) residencia en zona rural o urbana por un período menor a 2 años $(\mathrm{n}=47)$; (c) personas que presentaron problemas de salud mental y que no dieron el consentimiento informado $(n=2)$; (d) mujeres embarazadas $(\mathrm{n}=4), y(\mathrm{e})$ personas que rechazaron participar del estudio $(n=187)$.

El peso y la talla fueron determinados con una balanza electrónica (TANITA TBF 300A, USA) y tallímetro (SECA A800, USA) con precisión de 100 g y $1 \mathrm{~mm}$, respectivamente. El índice de masa corporal (IMC) fue calculado usando la ecuación de [peso/talla $\left.{ }^{2}\right]$ y el estado nutricional se definió mediante los valores de corte de la Organización Mundial de la Salud ${ }^{12}$. El perímetro de cintura fue medido con una cinta métrica no distendible (SECA, USA). Para definir obesidad central se utilizaron los puntos de corte propuestos por el Ministerio de Salud de Chile, basados en los parámetros para población del sudeste asiático, que corresponde a $\geq 90 \mathrm{~cm} \mathrm{y} \geq 80 \mathrm{~cm}$ en hombres y mujeres, respectivamente ${ }^{13}$. La composición corporal se determinó mediante la medición de 4 pliegues cutáneos (tricipital, bicipital, subescapular y suprailiaco) y el porcentaje de masa grasa se estimó mediante la ecuación de Durnin ${ }^{14}$.

Para la determinación de variables metabólicas se tomaron muestras de sangre tras un ayuno de 8 a 12 h. Para glicemia, colesterol total, triglicéridos (TG) y colesterol HDL (HDL-c) se utilizaron métodos enzimáticos colorimétricos ${ }^{8}$. El colesterol LDL (LDL-c) fue determinado con la ecuación de Friedewald ${ }^{15}$, la insulina fue determinada mediante radio-inmuno ensayo y la resistencia a la insulina mediante la ecuación de HOMA-IR ${ }^{16}$.

La presión arterial fue establecida como el promedio de dos mediciones realizadas después de estar 10 minutos en reposo, utilizando un monitor validado (OMRON HEM705, UK). Los datos socio-demográficos fueron recolectados mediante encuestas validadas.

El test de Chester fue utilizado para la medición del fitness cardiorrespiratorio (FCR) y fue expresado en METs ${ }^{17}$. Los niveles de AF y tiempo sedente fueron medidos con acelerómetros de movimiento (Actigraph GT1M, USA). El monitor se utilizó durante 7 días y sólo se removió durante las horas destinadas a dormir, 
ducharse o realización de actividades acuáticas. La intensidad de la AF (sedentaria, ligera, moderada y vigorosa) fue determinada mediante el algoritmo y categorías de Freedson ${ }^{18,19}$. El tiempo destinado a AF total se estimó mediante la suma del promedio de tiempo destinado a AF ligera, moderada y vigorosa, y fue expresada en MET/ $\mathrm{min} /$ día. La prevalencia de inactividad física se determinó utilizando el punto de corte de $<600$ $\mathrm{MET} / \mathrm{min} / \mathrm{semana}$ de AF de intensidad moderada o vigorosa. El sedentarismo se determinó utilizando el punto de corte de actividades $<1,5$ METs. El tiempo sedentario se presenta como la media de horas/día y se calcularon los terciles de sedentarismo para estratificar a la población como bajo ( $<8 \mathrm{~h} /$ día), medio (8-9,5 h/día) o alto nivel de sedentarismo $(>9,5 \mathrm{~h} /$ día $)$.

Los patrones alimenticios fueron evaluados mediante el registro y peso del consumo de alimentos durante 7 días, obteniéndose la información del consumo de nutrientes a partir de la base de datos chilena de composición nutricional de alimentos ingresados en el software MINUTA de la Universidad de Concepción ${ }^{8}$.

\section{Análisis de datos}

La distribución normal de las variables fue analizada mediante los tests de Anderson Darling y Kruskal-Wallis. Las diferencias entre individuos según terciles de sedentarismo para variables cuantitativas fueron estimadas mediante General Linear Model (GLM) y las diferencias en porcentajes entre grupos fueron estimadas mediante Chi-cuadrado. La relación entre tiempo sedentario, AF y consumo de macronutrientes fue determinada mediante el test de correlación de Pearson. La asociación entre $1 \mathrm{~h}$ de incremento en el tiempo destinado a actividades sedentarias, terciles de sedentarismo y variables de tipo cardiometabólicas fueron determinadas mediante regresión múltiple, ajustando los modelos estadísticos por variables confusoras (edad, género, nivel educacional, tabaquismo, ingesta energética total, consumo de grasas, consumo de alcohol). Las variables metabólicas fueron ajustadas por IMC. Los análisis de tendencia $(P$-trend) para terciles de sedentarismo fueron determinadas mediante regresión lineal múltiple. Valor $\mathrm{p}$ aceptado para diferencias significativas fue $<0,05$. Todos los análisis fueron realizados con el software STATA SE 14, USA.

\section{Resultados}

Al comparar los terciles de sedentarismo (Tabla 1$)$, los sujetos altamente sedentarios $(>9,5 \mathrm{~h} /$ día) presentaron mayor peso corporal, IMC, masa grasa corporal, perímetro de cintura, prevalencia de inactividad física y un menor fitness cardiorrespiratorio que el grupo menos sedentario $(<8 \mathrm{~h} /$ día). El grupo altamente sedente presentó mayor prevalencia de sobrepeso/obesidad (IMC $\geq 25,0$ ), obesidad central, tabaquismo; concentrando este grupo, el mayor porcentaje de personas con educación secundaria (Tabla 1). El grupo más sedentario concentró un mayor porcentaje de personas con educación secundaria. No se encontraron diferencias significativas para variables de alimentación, pero se observó una tendencia a un mayor consumo de grasas saturadas, grasas monoinsaturadas y consumo de sal (sodio) en el grupo con mayores niveles de sedentarismo en comparación al grupo menos sedentario.

Los niveles de sedentarismo variaron entre 5,5 a 12,1 h/día. En promedio se destinaban 8,7 $\mathrm{h}$ /día a actividades de tipo sedentarias (64,8\% del día sin incluir las horas de sueño) (Figura 1). Sólo $4,2 \%$ y $0,3 \%$ eran utilizados en AF de intensidad modera y vigorosa, respectivamente. Resultados estratificados por género se presentan en la Figura 1. En relación a las recomendaciones internacionales de AF ( $\geq 600 \mathrm{MET} / \mathrm{min} / \mathrm{semana}$ ), 33,8\% de la población estudiada no cumplía con esta recomendación, siendo las mujeres significativamente más inactivas que los hombres $(36,1 \%$ versus $30,6 \%$, respectivamente, $\mathrm{p}<0,0001$ ).

La relación de tiempo sedente con AF total y alimentación se presenta en la Figura 2. Se encontró una correlación negativa entre tiempo sedente y AF total $(\mathrm{r}=-0,626 ; \mathrm{p}<0,0001)$, no obstante, no se encontraron asociaciones entre el tiempo destinado a actividades sedentarias versus el consumo energético total, consumo de grasas totales, grasas saturadas, azúcares y alcohol.

En la Tabla 2 se observan los cambios experimentados en los factores de riesgo cardiovascular y metabólicos al incrementar los niveles de sedentarismo. Por cada hora de incremento en el tiempo de sedentarismo al día se observaron deterioros significativos en todos los factores de riesgo, independiente de factores confusores. Con el fin de comparar las diferencias en la magnitud del efecto de $1 \mathrm{~h}$ de incremento en tiempo de sedentarismo 
Tabla 1. Características socio-demográficas, antropométricas, de actividad física y alimentarias según terciles de sedentarismo

\begin{tabular}{|c|c|c|c|c|c|}
\hline & \multirow[b]{2}{*}{ Todos } & \multicolumn{3}{|c|}{ Terciles de sedentarismo } & \multirow[b]{2}{*}{ Valor $\mathbf{p}$} \\
\hline & & $\begin{array}{l}\text { Bajo } \\
\text { (< } 8 \text { h/día) }\end{array}$ & $\begin{array}{l}\text { Medio } \\
\text { (8-9,5 h/día) }\end{array}$ & $\begin{array}{l}\text { Alto } \\
\text { (> 9,5 h/día) }\end{array}$ & \\
\hline \multicolumn{6}{|l|}{ Socio-demográficos } \\
\hline$n$ & & 108 & 107 & 107 & \\
\hline Edad (años) & $37,6 \quad(12,9)$ & $36,7 \quad(12,6)$ & $35,3 \quad(11,5)$ & $40,7 \quad(13,9)$ & 0,064 \\
\hline Género (\% de mujeres) & 56,2 & 56,5 & 56,1 & 56,1 & 0,998 \\
\hline \multicolumn{6}{|l|}{ Educación (\%) } \\
\hline Básica & 27,9 & 34,9 & 27,4 & 21,5 & 0,039 \\
\hline Secundaria & 39,8 & 36,8 & 33,0 & 49,5 & \\
\hline Técnico/Universitaria & 32,2 & 28,3 & 39,6 & 29,0 & \\
\hline \multicolumn{6}{|l|}{ Tabaquismo } \\
\hline Fumador (\%) & 44,4 & 33,3 & 58,8 & 78,5 & $<0,0001$ \\
\hline \multicolumn{6}{|l|}{ Antropométricas } \\
\hline Peso corporal (kg) & $71,2 \quad(9,9)$ & $(8,6)$ & $71,9 \quad(9,7)$ & $74,3 \quad(10,2)$ & $<0,0001$ \\
\hline Talla $(\mathrm{cm})$ & $159,3 \quad(8,4)$ & $158,1 \quad(7,3)$ & $161,0 \quad(8,5)$ & $158,7 \quad(9,1)$ & 0,794 \\
\hline IMC $\left(\mathrm{kg} / \mathrm{m}^{-2}\right)$ & $28,1 \quad(3,4)$ & $(3,2)$ & $27,7 \quad(3,2)$ & $29,4 \quad(3,3)$ & $<0,0001$ \\
\hline \multicolumn{6}{|l|}{ Estado nutricional (\%) } \\
\hline Normal & 18,9 & 25,9 & 18,7 & 12,1 & $<0,0001$ \\
\hline Sobrepeso & 52,4 & 15,7 & 25,2 & 44,9 & \\
\hline Obeso & 28,5 & 58,3 & 56,1 & 43,0 & \\
\hline Masa grasa (\%) & $29,4 \quad(4,6)$ & $(5,3)$ & $29,2(4,3)$ & $(3,5)$ & $<0,0001$ \\
\hline Perímetro de cintura $(\mathrm{cm})$ & $97,4 \quad(10,5)$ & $94,7 \quad(9,6)$ & $95,7(9,6)$ & $101,7 \quad(11,0)$ & 0,001 \\
\hline Obesidad central (\%) & 83,3 & 84,2 & 82,2 & 94,4 & 0,018 \\
\hline \multicolumn{6}{|l|}{ Actividad física } \\
\hline Fitness cardiorrespiratorio (METs) & $13,1 \quad(3,4)$ & $14,5 \quad(3,2)$ & $13,4 \quad(3,2)$ & $11,3 \quad(2,8)$ & $<0,0001$ \\
\hline AF total (METs/min/día) & $904,7(289)$ & $1094,3(259)$ & $915,9(259)$ & $698,0(208)$ & $<0,0001$ \\
\hline AF ligera (min/día) & $248,5 \quad(84,1)$ & $294,5 \quad(84,1)$ & $250,7 \quad(74,8)$ & $199,9 \quad(64,6)$ & $<0,0001$ \\
\hline AF moderada (min/día) & $33,6 \quad(23,6)$ & $44,6 \quad(23,8)$ & $34,1 \quad(23,8)$ & $22,1 \quad(16,6)$ & $<0,0001$ \\
\hline AF vigorosa (min/día) & $2,63 \quad(4,8)$ & $3,63 \quad(6,2)$ & $2,95 \quad(4,7)$ & $1,29 \quad(2,5)$ & $<0,0001$ \\
\hline AF modera-vigorosa (METs/min/día) & $156,8(118,1)$ & $208,8(123,3)$ & $161,3(122,5)$ & $98,6 \quad(74,8)$ & $<0,0001$ \\
\hline Prevalencia de inactividad física (\%) & 33,8 & 12,2 & 30,4 & 59,2 & $<0,0001$ \\
\hline Sedentarismo (h/día) & $8,71 \quad(1,5)$ & $7,04 \quad(0,5)$ & $8,67 \quad(0,3)$ & $(9,3)$ & $<0,0001$ \\
\hline \multicolumn{6}{|l|}{ Alimentación } \\
\hline Energía total (Kcal/día) & $2.703 \quad(728)$ & $2.706 \quad(943)$ & $2.776,6(918)$ & $2.630 \quad(787)$ & 0,595 \\
\hline Proteína (\% de energía total) & $(4,2)$ & $15,1 \quad(4,6)$ & $13,8 \quad(3,9)$ & $(4,1)$ & 0,530 \\
\hline Carbohidratos (\% de energía total) & $(9,4)$ & $57,1 \quad(9,1)$ & $58,9 \quad(9,2)$ & $52,2 \quad(9,9)$ & 0,422 \\
\hline Grasas totales (\% de energía total) & $(8,3)$ & $(7,5)$ & $24,8 \quad(8,5)$ & $(8,8)$ & 0,283 \\
\hline Grasas saturadas (g/día) & $19,7 \quad(14,0)$ & $19,3 \quad(10,9)$ & $19,1 \quad(11,5)$ & $20,6 \quad(11,7)$ & 0,086 \\
\hline Grasas monoinsaturadas (g/día) & $24,0 \quad(14,1)$ & $23,1 \quad(13,3)$ & $23,8 \quad(15,0)$ & $25,1 \quad(13,9)$ & 0,055 \\
\hline Grasas poliinsaturadas (g/día) & $12,6 \quad(7,6)$ & $(6,8)$ & $12,9 \quad(7,6)$ & $(8,4)$ & 0,302 \\
\hline Azúcar (g/día) & $134,6 \quad(85,5)$ & $145,2 \quad(93,0)$ & $129,2 \quad(73,2)$ & $129,6 \quad(88,1)$ & 0,065 \\
\hline Sodio (g/día) & $3,5 \quad(2,9)$ & $(2,2)$ & $3,8 \quad(3,1)$ & $4,0 \quad(3,1)$ & 0,076 \\
\hline Fibra dietética (g/día) & $(3,5)$ & $5,3 \quad(3,5)$ & $(3,6)$ & $(3,3)$ & 0,666 \\
\hline Alcohol (\% de energía total) & $(2,4)$ & $2,1 \quad(2,2)$ & $(2,0)$ & $(2,8)$ & 0,548 \\
\hline
\end{tabular}

Datos presentados como media (desviación estándar) para variables continuas y como porcentajes para variables categóricas. En negrita se destacan los valores significativos $(p<0,05)$. 


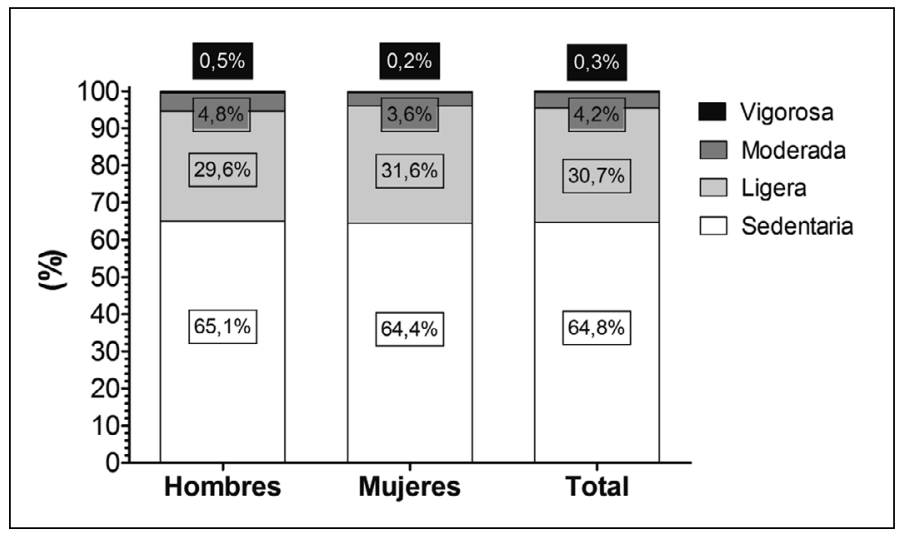

Figura 1. Distribución del tiempo diario destinado a diferentes intensidades de actividad física y tiempo sedente. Datos presentados como porcentaje del día sin considerar horas destinadas a dormir.

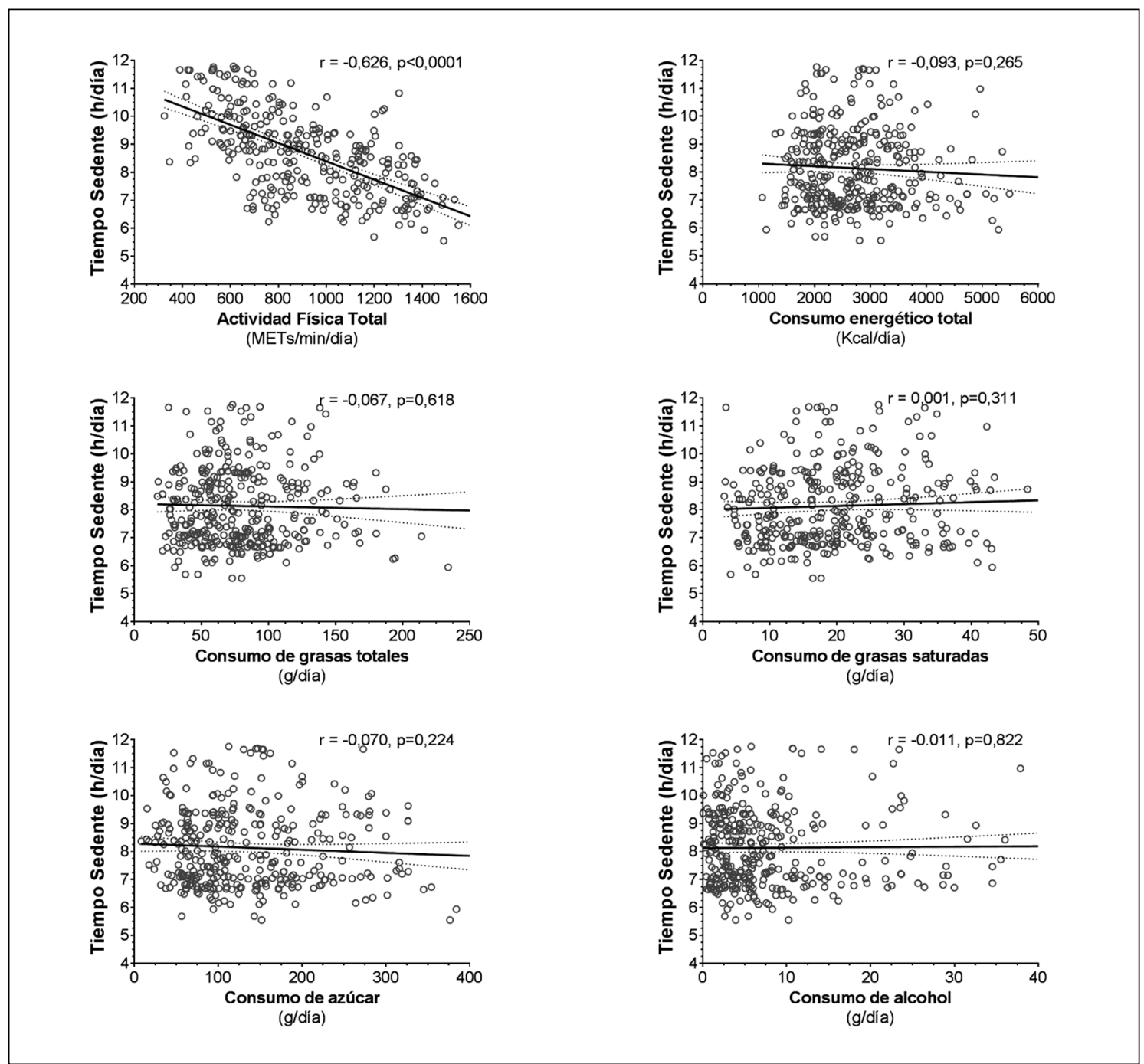

Figura 2. Correlación entre el tiempo sedente, actividad física total y consumo alimentario. Datos son presentados como coeficiente de correlación de Pearson (r). 
Tabla 2. Cambios en marcadores de riesgo cardiovascular y metabólicos asociados a 1 h de incremento al día en niveles de sedentarismo

\begin{tabular}{|c|c|c|c|}
\hline & Beta $(95 \%$ IC) & Beta estandarizado (95\% IC)* & Valor $\mathbf{p}$ \\
\hline $\mathrm{IMC}\left(\mathrm{m} / \mathrm{kg}^{-2}\right)$ & $0,69(0,45$ a 0,92$)$ & $0,18(0,12$ a 0,25$)$ & $<0,0001$ \\
\hline Perímetro de cintura $(\mathrm{cm})$ & $1,95(1,21$ a 2,69$)$ & $0,17(0,11$ a 0,24$)$ & $<0,0001$ \\
\hline Masa grasa (\%) & $1,03(0,71$ a 1,35$)$ & $0,22(0,15$ a 0,28$)$ & $<0,0001$ \\
\hline PAS $(\mathrm{mmHg})$ & $2,48(1,30$ a 3,66$)$ & $0,14(0,07$ a 0,21$)$ & $<0,0001$ \\
\hline $\mathrm{PAD}(\mathrm{mmHg})$ & $1,49(0,61$ a 2,37$)$ & $0,12(0,05$ a 0,19$)$ & 0,001 \\
\hline Glicemia (mg/dl) & $4,79(3,23$ a 6,35$)$ & $0,22(0,15$ a 0,29$)$ & $<0,0001$ \\
\hline Insulina (pmol/l) & $2,73(2,25$ a 3,22$)$ & $0,23(0,18$ a 0,26$)$ & $<0,0001$ \\
\hline HOMA-IR & $0,75(0,62$ a 0,87$)$ & $0,27(0,22$ a 0,30$)$ & $<0,0001$ \\
\hline Colesterol total (mg/dl) & $9,73(6,28$ a 13,1$)$ & $0,21(0,13$ a 0,28$)$ & $<0,0001$ \\
\hline HDL-c (mg/dl) & $-3,50(-4,57$ a $-2,43)$ & $-0,23(-0,31$ a $-0,16)$ & $<0,0001$ \\
\hline $\mathrm{LDL}-\mathrm{c}(\mathrm{mg} / \mathrm{dl})$ & $10,7(7,1$ a 14,4$)$ & $0,21(0,14$ a 0,29$)$ & $<0,0001$ \\
\hline $\mathrm{TG}(\mathrm{mg} / \mathrm{dl})$ & $12,4(8,44$ a 16,4$)$ & $0,22(0,14$ a 0,28$)$ & $<0,0001$ \\
\hline
\end{tabular}

Datos presentados como coeficiente beta y su respectivo $95 \%$ de intervalo de confianza por cada $1 \mathrm{~h} /$ día de incremento en actividades de tipo sedentarias. *Para los análisis de beta estandarizado, los resultados son presentados como cambios en las variables de interés expresados en desviación estándar por $1 \mathrm{~h}$ /día de incremento en tiempo sedente. Todos los análisis fueron ajustados por edad, sexo, educación, tabaquismo, actividad física total, tiempo de registro con acelerometría, ingesta calórica total, consumo total de grasas, alcohol, y azúcares. Variables metabólicas también fueron ajustadas por IMC. Abreviaciones: IMC: índice masa corporal; PAS: presión arterial sistólica; PAD: presión arterial diastólica; HOMA-IR: resistencia a la insulina; HDL-c: colesterol-HDL; LDL-c: colesterol-LDL; TG: triglicéridos.
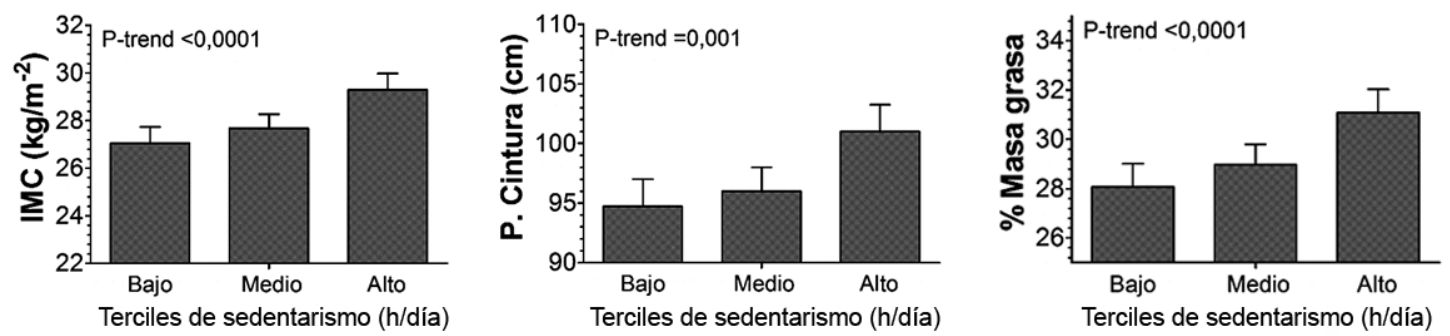

Figura 3. Asociación entre niveles de sedentarismo y marcadores de adiposidad. Datos presentados como promedio y error estándar de la media. El valor-p estimado representa la tendencia ( $P$-trend). Todos los análisis fueron ajustados por edad, sexo, educación, tabaquismo, actividad física total, tiempo de registro con acelerometría, ingesta calórica total, consumo de total de grasas, alcohol, y azúcares.

sobre los factores de riesgo, se estandarizaron a puntaje $\mathrm{Z}$ todas las variables dependientes (Beta estandarizado, Tabla 2). Estos resultados revelan que el sedentarismo afecta de manera más perjudicial a HOMA-IR, insulina, glicemia, porcentaje de masa grasa y perfil lipídico.

En las Figuras 3 y 4 se presentan los resultados para terciles de sedentarismo y su asociación con factores de riesgo cardiovascular y metabólicos. Estos resultados reflejan que el incremento en los terciles de sedentarismo se asocia a un aumento significativo de la adiposidad y deterioro significativo de la salud cardiovascular, independiente de factores socio-demográficos, alimenticios, IMC y AF. 

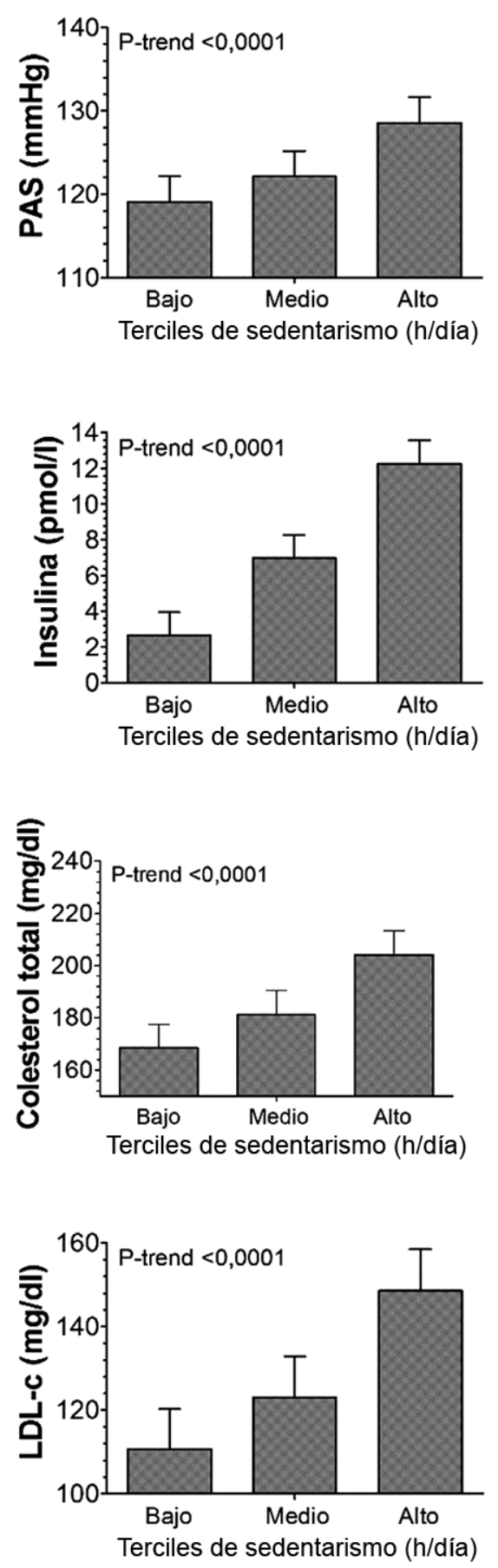
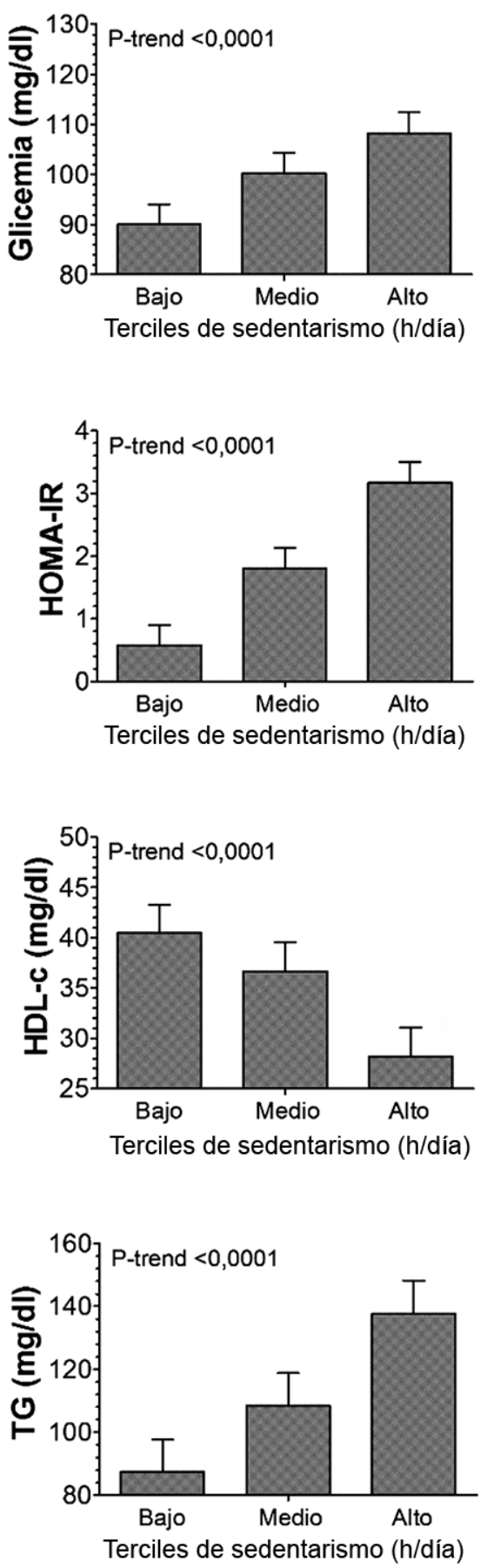

Figura 4. Asociación entre niveles de sedentarismo con factores de riesgo cardiovascular y metabólicos. Datos presentados como promedio y error estándar de la media. El valor-p estimado representa la tendencia $(P$-trend). Todos los análisis fueron ajustados por edad, sexo, educación, tabaquismo, actividad física total, tiempo de registro con acelerometría, ingesta calórica total, consumo total de grasas, alcohol, azúcares e IMC. 


\section{Discusión}

\section{Principales hallazgos}

El aumento del tiempo destinado a actividades sedentarias se correlaciona con un incremento en los factores de riesgo cardiovascular y metabólicos. El efecto del sedentarismo sobre estos marcadores fue independiente de factores socio-demográficos, alimentación, IMC y AF. Estos resultados son relevantes, ya que comprueban que el efecto del sedentarismo sobre estos factores de riesgo no estaría modulado por una mayor ingesta calórica, sino por un gasto energético reducido. Esto se debería al excesivo tiempo destinado a actividades sedentes, cuyo gasto energético es menor a $1,5 \mathrm{kcal} / \mathrm{kg} / \mathrm{h}^{11}$.

\section{Comparación y relación con estudios previos}

Los resultados encontrados en la presente investigación concuerdan con numerosos estudios que han sido resumidos en revisiones sistemáticas y metaanálisis recientes ${ }^{20-22}$. Brocklebank y cols., en una revisión sistemática, identificaron 28 estudios que reportaron asociación entre sedentarismo y factores de riesgo cardiovascular, donde los mayores efectos nocivos del sedentarismo fueron observados en marcadores de diabetes mellitus, incluyendo, glicemia, insulina y marcadores de resistencia a la insulina ${ }^{20}$. Por su parte, Wilmot y cols., en un metaanálisis encontraron que individuos con altos niveles de sedentarismo presentan un riesgo de desarrollar diabetes $112 \%$ mayor en comparación a aquellos que destinan menor tiempo a actividades sedentarias ${ }^{21}$.

Si bien se ha postulado que la relación entre sedentarismo y obesidad está principalmente asociada a un consumo excesivo de calorías y alimentos no saludables, como lo son grasas saturadas y azúcares ${ }^{23,24}$, nuestro estudio reveló que aquellos individuos con mayor tiempo de sedentarismo no presentan un patrón de alimentación significativamente diferente a aquellos con bajos niveles de sedentarismo. Sería interesante incorporar en futuros estudios la medición de subcomponentes de sedentarismo total como lo es ver televisión, para investigar si conductas sedentarias de tiempo libre podrían están relacionadas a una alimentación no saludable.

Al comparar los resultados de este estudio con los niveles de sedentarismo reportados por la Encuesta Nacional de Salud 2009-2010 mediante el uso de cuestionarios ${ }^{7}$, es interesante observar que hay una gran diferencia entre el promedio de tiempo sentado reportado al día (3,5 h/día) y los niveles de sedentarismo encontrados en el presente estudio, estimados mediante la utilización de acelerómetros de movimiento $(8,7 \mathrm{~h} /$ día). Estudios previos en población chilena han reportado que la medición de AF y sedentarismo mediante el uso de cuestionarios podría estar subestimando en aproximadamente $50 \%$ la relación existente entre los niveles de sedentarismo y marcadores de salud metabólica ${ }^{10}$; por lo cual, es importante considerar las limitaciones del uso de cuestionarios para la estimación de los niveles de sedentarismo y AF.

\section{Fortalezas y limitaciones del estudio}

Este estudio presenta fortalezas y debilidades que deben considerarse al interpretar las implicaciones de los resultados. Entre sus fortalezas se encuentra la medición objetiva de la AF y sedentarismo (acelerometría), lo cual permite reducir el sesgo asociado a datos recolectados a través de cuestionarios $^{10}$. Dentro de las limitaciones se debe considerar que el presente estudio no es representativo de la población nacional, por lo cual, futuros estudios, principalmente encuestas nacionales, deberían realizar un esfuerzo por incluir metodologías objetivas para la medición de la AF.

\section{Implicaciones de los resultados}

El sedentarismo es un factor de riesgo para la salud cardiovascular y metabólica, que actúa independiente de los niveles de AF de un individuo. Esto reafirma la importancia de cumplir con las recomendaciones de AF para controlar y revertir el riesgo de desarrollo de enfermedades, pero a la vez, constituye una alerta, ya que los efectos beneficiosos de la AF podrían verse mermados por un exceso de tiempo sedentario ${ }^{3,11,25,26}$.

A la luz de los resultados obtenidos en el presente estudio y tomando en cuenta que gran parte de la población chilena realiza trabajos o actividades en tiempo libre que involucran una gran cantidad de tiempo sedente (ej., trabajo de oficina, trabajos frente a un computador, ver televisión, etc.), se hace imprescindible que futuras intervenciones y políticas públicas (a nivel de salud, educación, transporte, etc.) centren sus esfuerzos no sólo en incrementar los niveles de AF de los chilenos sino también en reducir los niveles de sedentarismo. 


\section{Conclusiones}

Altos niveles de sedentarismo tienen un efecto nocivo sobre factores de riesgo cardiovascular y metabólicos, asociados a la obesidad. Disminuir el tiempo destinado a actividades de bajo costo energético, como pasar mucho tiempo sentado, debiera ser una recomendación indispensable en las futuras guías de AF para la población.

Agradecimientos: Se agradece de forma especial a los doctores: Francisco Pérez, Natalia Ulloa y Carlos Calvo por su contribución en la recolección de datos de ese estudio; y a los doctores Jason Gill y Mark Bailey por su contribución en el diseño.

\section{Referencias}

1. Owen N, Healy GN, Matthews CE, Dunstan, DW. Too Much Sitting: The Population Health Science of Sedentary Behavior. Exerc Sport Sci Rev 2010; 38 (3): 105-13.

2. Dunstan DW, Howard B, Healy GN, Owen N. Too much sitting-A health hazard. Diabetes Res Clin Pract 2012; 97 (3): 368-76.

3. Cristi-Montero C, Celis-Morales C, Ramírez-Campillo R, Aguilar-Farías N, Álvarez C, Rodríguez-Rodríguez F. [Sedentary behaviour and physical inactivity is not the same!: An update of concepts oriented towards the prescription of physical exercise for health]. Rev Med Chile 2015; 143 (8): 1089-90.

4. Matthews CE, Chen KY, Freedson PS, Buchowski MS, Beech BM, Pate RR, et al. Amount of time spent in sedentary behaviors in the united states, 2003-2004. Am J Epidemiol 2008; 167 (7): 875-81.

5. Colley RC, Garriguet D, Janssen I, Craig CL, Clarke J, Tremblay MS. Physical activity of Canadian adults: accelerometer results from the 2007 to 2009 Canadian Health Measures Survey. Health Rep 2011; 22 (1): 7-14.

6. Bennie JA, Chau JY, van der Ploeg HP, Stamatakis E, Do A, Bauman A. The prevalence and correlates of sitting in European adults-a comparison of 32 Eurobarometer-participating countries. Int J Behav Nutr Phys Act 2013; 10: 107.

7. Celis-Morales C, Salas C, Alduhishy A, Sanzana R, Martínez MA, Leiva A, et al. Socio-demographic patterns of physical activity and sedentary behaviour in Chile: results from the National Health Survey 2009-2010. J Public Health 2016; 38 (2): e98-e105.

8. Celis-Morales CA, Pérez-Bravo F, Ibañes L, Sanzana
R, Hormazábal E, Ulloa N, et al. Insulin Resistance in Chileans of European and Indigenous Descent: Evidence for an Ethnicity x Environment Interaction. PLoS One 2011; 6 (9): e24690.

9. Celis-Morales C, Salas C, Álvarez C, Aguilar-Farías N, Ramírez-Campillo R, Leppe J, et al. [Higher physical activity levels are associated with lower prevalence of cardiovascular risk factors in Chile]. Rev Med Chile 2015; 143 (11): 1435-43.

10. Celis-Morales CA, Pérez-Bravo F, Ibáñez L, Salas C, Bailey MES, Gill JMR. Objective vs. self-reported physical activity and sedentary time: effects of measurement method on relationships with risk biomarkers. PloS one 2012; 7 (5): e36345.

11. Cristi-Montero C. Considerations regarding the use of metabolic equivalents when prescribing exercise for health: preventive medicine in practice. Physician Sportsmed 2016; 44 (2): 109-11.

12. WHO. Obesity: preventing and managing the global epidemic. Report of a WHO consultation. World Health Organization technical report series 2000; 894: i-xii, $1-253$.

13. Ministerio de Salud de Chile, MINSAL. Enfoque de riesgo para la prevención de enfermedades cardiovasculares. Consenso 2014.

14. Durnin JVGA, Womersley J. Body fat assessed for, total-body density and its estimation from skinfold thickness: measurement on 481 men and women aged from 16 to 72 years. Br J Nutr 1974; 32 (1): 77-97.

15. Friedewald WT, Levy RI, Fredrickson DS. Estimation of concentration of low-density lipoprotein cholesterol in plasma, without use of preparative ultracentrifuge. Clin Chem 1972; 18 (6): 499-502.

16. Matthews DR, Hosker JP, Rudenski AS, Naylor BA, Treacher DF, Turner RC. Homeostasis model assessment: insulin resistance and beta-cell function from fasting plasma glucose and insulin concentrations in man. Diabetologia 1985; 28 (7): 412-9.

17. Buckley JP, Sim J, Eston RG, Fox HR. Reliability and validity of measures taken during the Chester step test to predict aerobic power and to prescribe aerobic exercise. Br J Sports Med 2004; 38 (2): 197-205.

18. Freedson P, Bowles HR, Troiano R, Haskell W. Assessment of physical activity using wearable monitors: recommendations for monitor calibration and use in the field. Med Sci Sports Exerc 2012; 44 (1 Suppl 1): S1-4.

19. Freedson PS, Melanson E, Sirard J. Calibration of the Computer Science and Applications, Inc. accelerometer. Med Sci Sports Exerc 1998; 30 (5): 777-81.

20. Brocklebank L, Falconer C, Page A, Perry R, Cooper AR. Accelerometer-measured sedentary time and cardiome- 
tabolic biomarkers: A systematic review. Prev Med 2015; 76: 92-102.

21. Wilmot EG, Edwardson CL, Achana FA, Davies MJ, Gorely T, Gray LJ, et al. Sedentary time in adults and the association with diabetes, cardiovascular disease and death: systematic review and meta-analysis. Diabetologia. 2012; 55 (11): 2895-905.

22. Henson J, Yates T, Biddle SJ, Edwardson CL, Khunti K, Wilmot EG, et al. Associations of objectively measured sedentary behaviour and physical activity with markers of cardiometabolic health. Diabetologia 2013; 56 (5): 1012-20.

23. Sisson SB, Broyles ST, Robledo C, Boeckman L, Leyva $\mathrm{M}$. Television viewing and variations in energy intake in adults and children in the USA. Public Health Nutr 2012; 15 (4): 609-17.

24. Grontved A, Hu FB. Television Viewing and Risk of Type 2 Diabetes, Cardiovascular Disease, and All-Cause Mortality A Meta-analysis. JAMA 2011; 305 (23): 244855.

25. Cristi-Montero C, Rodríguez FR. [The paradox of being physically active but sedentary or sedentary but physically active]. Rev Med Chile 2014; 142 (1): 72-8.

26. Kulinski JP, Khera A, Ayers CR, Das SR, de Lemos JA, Blair SN, et al. Association Between Cardiorespiratory Fitness and Accelerometer-Derived Physical Activity and Sedentary Time in the General Population. Mayo Clin Proc 2014; 89 (8): 1063-71. 